BULL. AUSTRAL. MATH. SOC.

VOL. 23 (1981), 199-208.

\title{
NOTIONS OF TOPOS
}

\section{Ross StReET}

\begin{abstract}
A Grothendieck topos has the property that its Yoneda embediing has a left-exact left adjoint. A category with the latter property is called lex-total. It is proved here that every lextotal category is equivalent to its category of canonical sheaves. An unpublished proof due to Peter Freyd is extended slightly to yield that a lex-total category, which has a set of objects of cardinality at most that of the universe such that each object in the category is a quotient of an object from that set, is necessarily a Grothendieck topos.
\end{abstract}

Lex-total categories were introduced in [7, p. 377] and were shown to include Grothendieck toposes. These notions require the specification of a strongly inaccessible cardinal $\boldsymbol{N}$. A set is called small when it has cardinality less than $\aleph$, and is called moderate when it has cardinality less than or equal to $\$$. (Note that "small" may mean "finite" and "moderate" then means "countable".) Let $S$ denote the category of small sets.

A category $E$ is lex-total when $E$ has small homsets and the Yoneda embedding $y: E \rightarrow\left[E^{\mathrm{Op}}, S\right]$ has a left adjoint $z$ which preserves finite limits. Part of a theorem of Giraud [3, p. 303] states that a category $E$ is a Grothendieck topos if and only if $E$ has small homsets, every $S$-valued canonical sheaf on $E$ is representable, and $E$ has a small generating set of objects. (Recall that the canonical topology on a

Received 6 October 1980. I am grateful to Bob Walters and Murray Adelman for encouraging conversations on this material. 
category is the largest topology for which the representable functors are sheaves; canonical sheaves are sheaves for the canonical topology.)

The purpose of this article is to consider some relationships among the following conditions on a category $E$ with small homsets:

(1) $E$ is a Grothendieck topos;

(2) $E$ is lex total;

(3) every $S$-valued canonical sheaf on $E$ is representable and $E$ has all small colimits;

(4) $E$ is an elementary topos with all small colimits;

(5) $E$ is a pretopos with all small coproducts and the latter are preserved by pullback.

(For the definitions of "elementary topos" and "pretopos", see [4, p. 23, p. 238].)

The following implications hold:

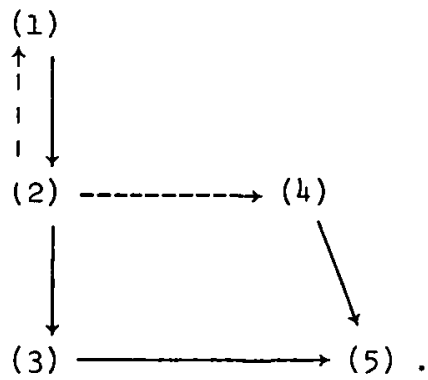

If $E$ has a generating small set of objects all the conditions (1)-(5) are equivalent. The dotted implications in the above diagram hold under a size condition apparently weaker than requiring a generating small set.

$(1) \rightarrow(2)$ was proved in [7] where it was also asserted that $(2) \rightarrow(4)$ provided $E$ is well-powered.

$(4) \rightarrow(5)$ is well-known elementary topos theory.

$(3)+(5)$ is easy.

$(2) \rightarrow(3)$ is Theorem A below.

$(2) \rightarrow(1)$, under the assumption that $E$ has a moderate set of isomorphism classes of objects, was proved by Peter Freyd in correspondence 
with the author. The proof of Theorem B below is essentially Freyd's proof modified to accommodate an apparently weaker assumption on $E$.

An example is given below to show that (4) does not imply (3).

André Joyal has suggested that (5) is a good notion of topos. Small generating sets may not exist in such categories (see the false toposes of [3, Exposé IV (2.8)]). Even if they do, they are not to be discovered canonically: witness the proof of Theorem $B$ which leans heavily on the axiom of chojce and proof by contradiction.

It is possible that there are lex-total categories (with very large sets of isomorphism classes) which are not Grothendieck toposes. (An example is not known to the author.) Even if this should be the case, it is the contention of Bob Walters and the author that lex-total categories are the good notion of topos. After all, what one expects of a topos is that it should be a category of sheaves on some site. The only canonical choice of site is the topos itself with the canonical topology. Theorem A shows that lex total categories meet this criterion. Another important property of lex-total categories is that colimit-preserving functors out of them have right adjoints [7, p. 372]; this property, which takes the place of the "generator" condition for many purposes, is not obviously satisfied by categories for which (3) holds.

Several years ago the author conjectured that (3) implies ( 1 ). However, we are unable to prove that (3) implies (2) even under the assumption that $E$ is well-powered.

The notion of lex-totality relative to a given size structure makes sense in a very general setting and yields important concepts in other particular cases. For example, for an elementary topos $B$, there is a size structure on the bicategory of fibrations over $B$ such that every elementary topos with generators (in the internal sense [4]) over $B$ gives rise to a lex-total object of the bicategory [5]. All the good properties of elementary toposes with generators over $B$ are shared by the lex-total objects. It seems unlikely, in the absence of the axiom of choice in $B$ that lex-total objects should all arise in this way.

Suppose $X$ is a category with pullbacks. For any family $C$ of arrows into some object $u$ of $X$, let $D(C)$ denote the free category on the graph described as follows. There are objects, $\xi$ and objects $(\xi, \eta)$ 
where $\xi, \eta$ are members of $C$. For each $\xi, \eta$ in $C$, there are arrows as shown below:

$$
\xi+(\xi, n) \rightarrow n
$$

There is a functor $k_{C}: D(C) \rightarrow X$ which takes the above span to the pullback of $\xi, n$ :

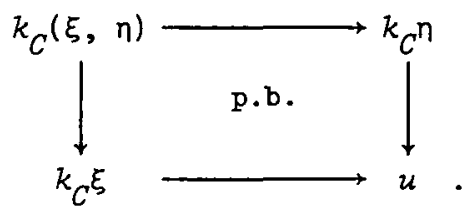

The arrows $\xi: k_{C} \rightarrow u$ are the components of a cocone $\lambda_{C}: k_{C} \rightarrow u$ with vertex $u$. A functor $f: X^{0 p} \rightarrow Z$ is a sheaf for a topology on $X$ when, for each covering family $c$ of arrows into $u$, the cocone $\lambda_{C}$ is taken by $f$ to a limit cone with vertex $f u$. The representable functors are all sheaves precisely when the cocone $\lambda_{C}$ is a colimit cocone for all covering families $C$. It follows easily that, for any topology for which the representables are sheaves, all covering families must be extremal epimorphic (terminology of [2, p. 177]).

THEOREM A. Every S-valued canonical sheaf on a lex-total category $E$ is representable.

- Proof. First we shall show that the canonical topology on $E$ consists of the extremal epimorphic families. Since the representables are to be sheaves, all covering families must be extremal epimorphic. Since pulling back has a right adjoint for a lex-total category $E$, the extremal epimorphic families do form a topology on $E$. It remains to show that the representables are sheaves for this topology. Let $C$ be an extremal epimorphic family of arrows into $u$. In $\left[E^{\mathrm{Op}}, S\right]$ the cocone $y \lambda_{C}: y k_{C} \rightarrow y u$ factors as a a composite $y k_{C} \rightarrow p \rightarrow y u$ where $y k_{C} \rightarrow p$ is a colimit cocone and $p \rightarrow y u$ is a monomorphism. Since $z$ preserves all colimits, preserves pullbacks, and has $z y=1$, we obtain a factorization of $\lambda_{C}$ as $k_{C} \rightarrow z p \rightarrow u$ where $k_{C} \rightarrow z p$ is a colimit and $z p \rightarrow u$ is a monomorphism. Since $k_{C} \rightarrow u$ is extremal epimorphic, $z p \rightarrow u$ is also 
extremal epimorphic and hence an isomorphism. So $\lambda_{C}: k_{C} \rightarrow u$ is a colimit cocone as required.

Let $S^{\prime}$ denote a category of sets such that $S$ and $E$ are categories in $S^{\prime}$. Write $\tilde{E}$ for the category of canonical $S$-valued sheaves on $E$, and write $\tilde{E}^{\prime}$ for the category of canonical $S^{\prime}$-valued sheaves on $E$. We have the diagram

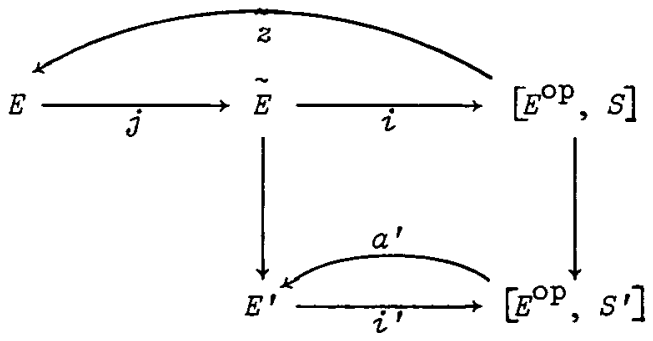

where $i, i^{\prime}$ and the vertical functors are inclusions, $i j=y$, and $a^{\prime}$ is the associated sheaf functor [1, p. 24].

For each extremal epimorphic family $c$, the functor $j$ preserves the colimit $\lambda_{C}: k_{C} \rightarrow u$. To see this, take $f \in \tilde{E}$. We must prove that $\tilde{E}\left(y \lambda_{C}, 1\right): \tilde{E}(y u, f) \rightarrow \tilde{E}\left(y k_{C}, f\right)$ is a limit cone. But, by Yoneda's lemma, the latter cone is isomorphic to $f \lambda_{C}: f u \rightarrow f k_{C}$ which is a limit cone since $f$ is a sheaf.

In order to prove the theorem we must show that, for each $f \in \tilde{E}$, the unit $f \rightarrow y z f$ is invertible.

Certainly the arrows $\sigma: y u \rightarrow f$ from representables form an epimorphic family $C$ in $\left[E^{\mathrm{op}}, S\right]$. So $\lambda_{C}: k_{C}+f$ is a colimit cocone in $\left[E^{\mathrm{Op}}, S\right]$ and also in $\left[E^{\mathrm{OP}}, S^{\prime}\right]$. Since $a^{\prime}$ preserves colimits, pullbacks, and has $a^{\prime} i^{\prime}=1$, we see that $\lambda_{C}: k_{C} \rightarrow f$ is a colimit cocone in $E^{\prime}$. Since the cocone is in $E$, it is a colimit in $E$.

Applying $z$ to the colimit $\lambda_{C}: k_{C} \rightarrow f$ in $\left[E^{\mathrm{op}}, S\right]$, we obtain a colimit cocone $z \lambda_{C}: z k_{C} \rightarrow z f$ which, since $z$ preserves pullbacks, is non-other than $\lambda_{M}: k_{M}+z f$ where $M$ is the family of all arrows into 
zf in $E$. Since $j$ preserves such colimits, we obtain a colimit $y \lambda_{M}: y k_{M} \rightarrow y z f$ in $\tilde{E}$.

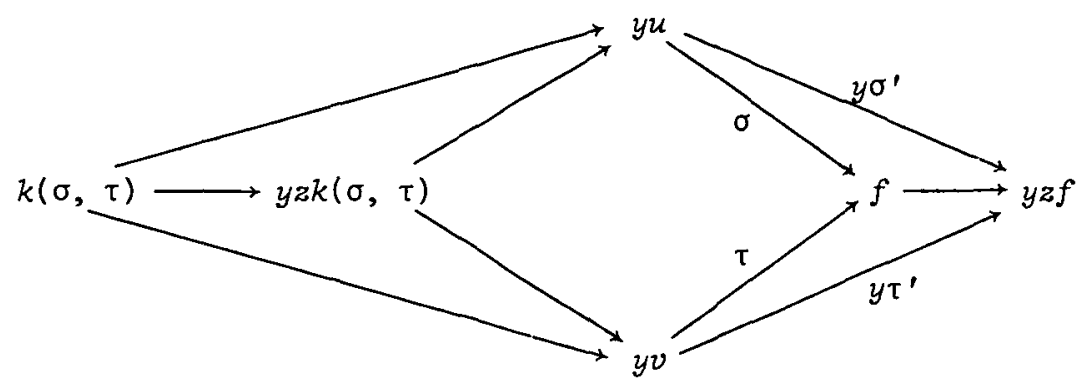

In the above diagram $k(\sigma, \tau)$ is the pullback of $\sigma, \tau$ and $y z k(\sigma, \tau)$ is the pullback of $y \sigma^{\prime}, y \tau^{\prime}$. Moreover, $f$ is a colimit over all $\sigma, \tau$ of the diagrams involving $k(\sigma, \tau), y u, y v$; while $y z f$ is a colimit over all $\sigma^{\prime}, \tau^{\prime}$ of the diagrams involving $y z k(\sigma, \tau), y u, y v$.

Consider now the special case where $f$ is a subobject of a representable. Then $f \rightarrow y z f$ is monomorphic; so, in the above diagram, $k(\sigma, \tau) \rightarrow y z k(\sigma, \tau)$ is invertible. Thus $f, y z f$ are colimits of isomorphic diagrams and hence $f \rightarrow y z f$ is invertible.

Return now to the general case. Since $k(\sigma, \tau)$ is a subobject of $y(u \times v)$, the arrow $k(\sigma, \tau)+y z k(\sigma, \tau)$ is invertible. So again, $f, y z f$ are colimits of isomorphic diagrams. So $f+y z f$ is invertible.

The proof of the following result is a mild modification of a proof of Peter Freyd.

THEOREM B. Suppose $E$ is a total category satisfying the following two conditions :

(a) if a pushout of a monomorphism is an isomorphism then the monomorphism is an isomorphism;

(b) there exists a moderate set $M$ of objects of $E$ such that, for each object $u$ of $F$, there exists an extremat epimorphism $v \rightarrow u$ with $v$ in $M$.

Then $E$ has a strongly generating small set of objects.

Proof. Suppose $E$ has no strongly generating small set of objects. Then $M$ is not small. We may assume $E$ is skeletal. Well order the 
objects of $M$ so that each $\{v \in M \mid v \leq u\}$ is small for each $u$ in $M$. The latter set cannot strongly generate $E$. So there exists a monomorphism $\mu_{u}: m_{u} \rightarrow n_{u}$ in $E$ which is not an isomorphism and yet $E\left(v, \mu_{u}\right): E\left(v, m_{u}\right)+E\left(v, n_{u}\right)$ is an isomorphism for all $v \leq u$. In $\left[E^{\mathrm{OP}}, S^{\prime}\right]$, form the colimit:

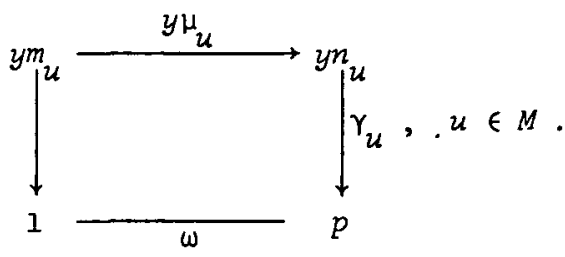

More explicitly, for $w \in E, p w$ is the disjoint union of 1 and, for each $u \in M$, the set of arrows $w \rightarrow n_{u}$ which do not factor through $\mu_{u}$. Using (b), we have an extremal epimorphism $v \rightarrow w$ with $v$ in $M$. It follows that $p w+p v$ is a monomorphism. Since $E\left(v, \mu_{u}\right)=\left(y \mu_{u}\right) v$ is an isomorphism for all $u \geq v$, it follows that $p v$ (and hence $p w$ ) is small. So $p$ lands in $S$. So the above diagram is a colimit in $\left[E^{\mathrm{op}}, S\right]$. Apply $z$ to obtain a colimit:

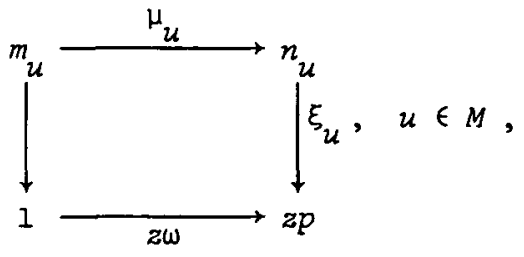

in $E$.

For each object $x$ of $M$, define $\varepsilon_{x}: z p \rightarrow z p$ by $\varepsilon_{x} \cdot z \omega=z \omega$, $\varepsilon_{x} \xi_{u}=\xi_{u}$ when $u=x$, and $\varepsilon_{x} \xi_{x}$ is $n_{u}+1 \underset{z \omega}{z} z p$ when $u \neq x$.

We next prove that the assignment $x \mapsto \varepsilon_{x}$ is a monomorphism from $M$ to $E(z p, z p)$. Suppose $\varepsilon_{x}=\varepsilon_{x^{\prime}}$ and $x \neq x^{\prime}$. Then $\varepsilon_{x} \xi_{x}=\varepsilon_{x^{\prime}} \xi_{x}$. So $\xi_{x}$ factors through $z \omega$. It follows from the above colimit property of $z p$ that the diagram below is a pushout: 


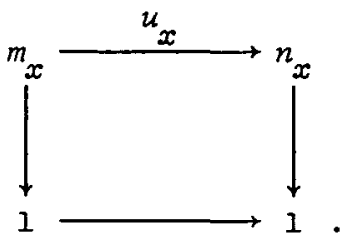

Using (a), we obtain that $\mu_{x}$ is an isomorphism, a contradiction.

However, $E(z p, z p)$ is a small set. So $M$ is small, a contradiction.

In a lex-total category, a pushout of a monomorphism is automatically a pullback, so condition (a) holds.

COROLLARY. A lex-total category $E$ is a Grothendieck topos if and only if it satisfies condition (b) of Theorem $B$.

EXAMPLE. The "false toposes" of [3] provide examples of categories $E$ with small homsets which satisfy conditions (4), (5), and condition (b) of Theorem B, and yet do not satisfy conditions (1), (2), (3).

To fix ideas we suppose $N$ to be countable so that $S$ is the category of finite sets. Take $G$ to be the infinite cyclic group and $E$ to be the category of finite $G$-sets. Then $E$ satisfies (4), (5) and has a moderate set of isomorphism classes. An object of $E$ can be regarded as a finite set with a permutation on it. An atom of $E$ is an object with. no proper subobjects. Notice that there are atoms of all small cardinalities (take the cycles!).

The covers for the canonical topology on $E$ are the epimorphic families. Let $A$ denote a set of representatives for the isomorphism classes of atoms! Each object of $E$ can be covered by a family of disjoint monomorphisms from objects of $A$. The topology on $A$ (regarded as a full subcategory of $E$ ), induced by canonical topology of $E$, is generated by covers consisting of single arrows. (Every arrow in $A$ is an epimorphism in $E$.$) By the comparison lemma [8, p. 288], each sheaf on A$ has a unique (up to isomorphism) extension to a sheaf on $E$. Let $f: A^{\text {op }} \rightarrow S$ be the coproduct of all the representables $A(-, a), a \in A$; note that $f$ lands in $S$ since each $b \in A$ has an arrow into only a small set of $a \in A$. It is easily seen that $f$ is a sheaf, and so 
extends to a sheaf $g: E^{\mathrm{OP}} \rightarrow S$.

We claim $g$ is not representable. Suppose $g$ is represented by some $x \in E$. Then, for each $b \in A$, we have $E(b, x) \cong g b=f b \cong \sum_{a \in A} E(b, a)$. It follows easily that, for each $a \in A$, there is a monomorphism $a+x$ which is impossible.

Thus $E$ does not satisfy (3).

\section{References}

[1] M. Artin, Grothendieck topologies (Department of Mathematics, Harvard University, Massachusetts, 1962).

[2] P.J. Freyd and G.M. Kelly, "Categories of continuous functors, I", $J$. Pure App 2. Algebra 2 (1972), 169-191.

[3] A. Grothendieck et J.L. Verdier, "Topos", Théorie des topos et cohomologie etale des schémas, Expose IV, 299-519 (Séminaire de Géométrie Algébrique du Bois Marie, 1963/64. Lecture Notes in Mathematics, 269. Springer-Verlag, Berlin, Heidelberg, New York, 1972).

[4] P.T. Johnstone, Topos theory (London Mathematical Society Monographs, 10. Academic Press, London, New York, San Francisco, 1977).

[5] Ross Street, "Cosmoi of internal categories", Trans. Amer. Math. Soc. 258 (1980), 271-318.

[6] Ross Street, "Extract from a letter on legitimacy of categories of presheaves", Diagrames (to appear).

[7] Ross Street and Robert Walters, "Yoneda structures on 2-categories", J. AZgebra 50 (1978), 350-379. 
[8] J.L. Verdier, "Fonctorialite des categories de faisceaux", Théorie des topos et cohomologie etale des schémas, Exposé III, 265-297 (Séminaire de Géomêtrie Algébrique du Bois Marie, 1963/64. Lecture Notes in Mathematics, 269. Springer-Verlag, Berlin, Heidelberg, New York, 1972).

School of Mathematics and Physics, Macquarie University, North Ryde, New South Wales 2113, Australia. 